\title{
High dynamic range imaging method for interferometry
}

\author{
J. Vargas ${ }^{\text {a,* }}$, R. Restrepo a , J. Antonio Quiroga ${ }^{\text {b }}$, T. Belenguer ${ }^{\text {a }}$ \\ a Laboratorio de Instrumentación Espacial, Instituto Nacional de Técnica Aeroespacial, Carretera de Ajalvir Km 4, 28850, Torrejón de Ardoz (Madrid), Spain \\ b Optics Department, Universidad Complutense de Madrid, Facultad de CC. Físicas, Ciudad Universitaria s/n, 28040 Madrid, Spain
}

\section{A R T I C L E I N F O}

\section{Article history:}

Received 28 February 2011

Received in revised form 4 April 2011

Accepted 25 April 2011

Available online 12 May 2011

\section{Keywords:}

High dynamic range imaging

Interferometry

Fringe analysis

\begin{abstract}
A B S T R A C T
We demonstrate a method to easily and quickly extend the dynamic range imaging capabilities of the camera in a typical interferometric approach. The camera dynamic range is usually low and limited to 256 gray levels. Also, it is well known that one may have over or under-exposed regions in the interferogram (due to nonuniform illumination) which makes these image regions not reliable. In our proposed method it is not necessary to obtain or use the non-linear camera response curve in order to extend the camera dynamic range. We obtain a sequence of differently exposed interferograms, typically five or six; after that, we compute the corresponding normalized fringe patterns and modulation maps using a typical normalization method. These normalized patterns are combined through a temporal weighted average using as weights the corresponding modulation maps. We show a set of experimental results that prove the effectiveness of the proposed method.
\end{abstract}

(c) 2011 Elsevier B.V. All rights reserved.

\section{Introduction}

The demodulation of fringe patterns is an interesting problem that has many applications [1]. These algorithms aim to extract the modulating phase from one or several fringe patterns. A typical preprocessing step is the normalization of the fringe pattern or patterns that consist in background suppression and modulation equalization so the processed fringe pattern has no DC or background component and its modulation map is constant over the whole fringe pattern. In expression (1) we show a typical fringe pattern,

$I(x, y)=I_{0}(x, y)+I_{m}(x, y) \cos (\Phi(x, y))$

where $I_{0}(x, y)$ and $I_{m}(x, y)$ are the DC and the modulation maps respectively and $\Phi(x, y)$ is the modulating phase map. The normalized version of expression (1) is given in (2)

$I_{n}(x, y)=\cos (\Phi(x, y))$

Typically, normalization is necessary in several fringe pattern processing methods as regularization and fringe direction determination methods. In this work, normalization is used as a pre-processing

\footnotetext{
* Corresponding author. Tel.: + 34915206382.

E-mail address: jvargas@fis.ucm.es (J. Vargas).
}

step that allows us to conveniently combine differently exposed interferograms. Our challenge is to obtain a reliable fringe pattern from a set of differently exposed fringe patterns. The different acquired fringe patterns are characterized by a high varying modulation map so there are bright and dark regions in the same fringe pattern map. This problematic is very common in interferometry, where typically two Gaussian beams are superimposed. The resultant interferogram has a bright central part and dark borders. Because of the limited camera dynamic range, it is not possible to capture the information from the bright and dark regions using a single camera exposure time. If we select the camera exposure time to correctly capture the dark zones then the details in the bright regions are lost by overexposure. Likewise, if the integration time is set to capture the bright zones correctly, then the underexposed dark regions are lost in sensor noise. Therefore, in order to extend the dynamic range of the imaging device it is necessary to mix differently exposed images.

Standard high dynamic range imaging methods are based on first obtaining the imaging sensor response function up a scale factor through a calibration process [2-5]. Once the sensor response function is determined, a high dynamic range (HDR) image, in which the pixel values are proportional to the true radiance values is computed. In order to determine the sensor response curve the calibration algorithm requires a set of differently exposed images of a static scene. The camera exposure times of the different images are also required. The calibration algorithm is based on a regularization method that permits obtaining the sensor response function in solving a leastsquares problem. Additionally, the resultant HDR pattern is affected by a large varying modulation map [4] so typically it is necessary to carry out an additional normalization process. 
(a)

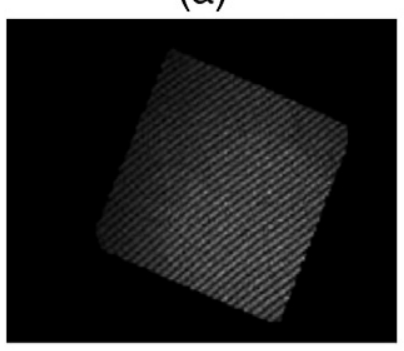

(d)
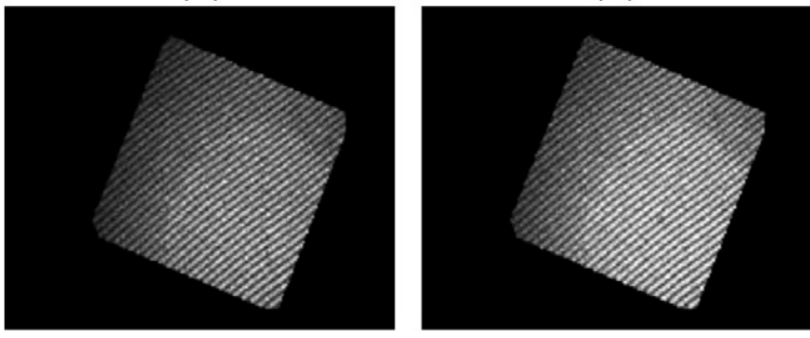

(c)

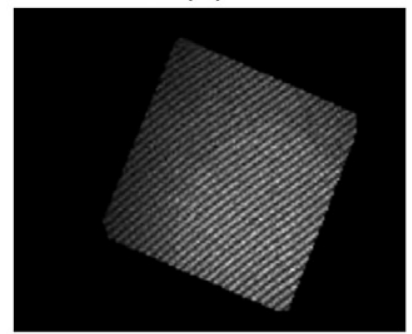

(f)

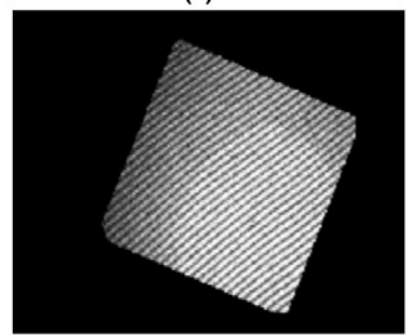

Fig. 1. Differently exposed open fringe pattern images used in the first experiment

(a)

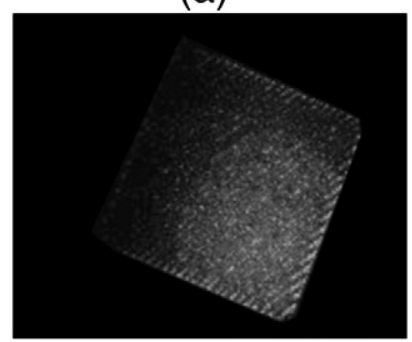

(d)

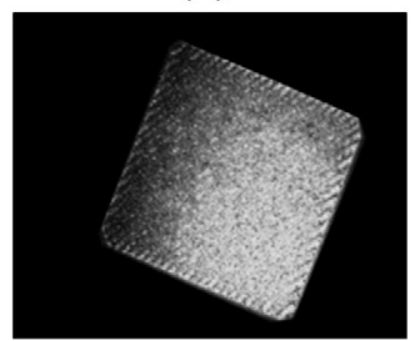

(b)

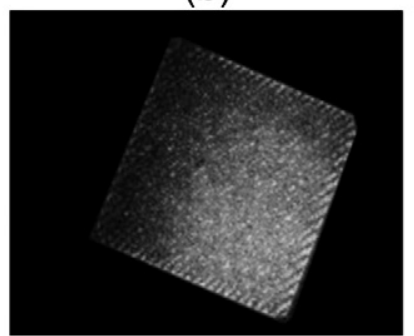

(e)

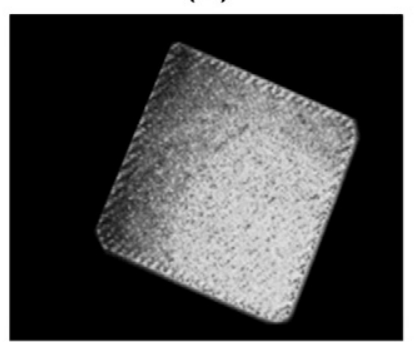

(c)

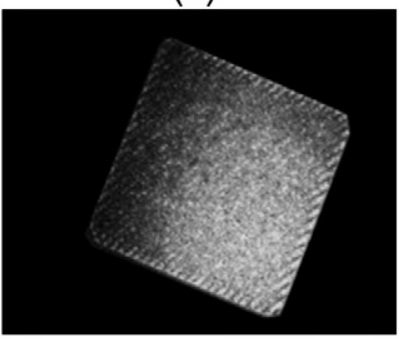

(f)

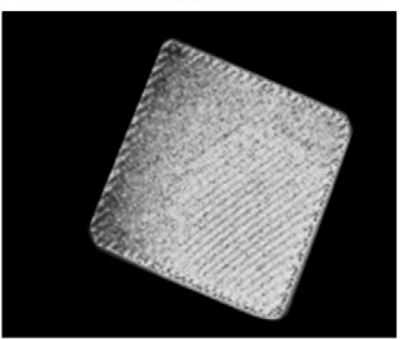

Fig. 2. Modulation maps corresponding to the fringe patterns shown in Fig. 1.

In this work we propose a HDR method specially designed for interferometry. The method obtains a reliable fringe pattern without the need of any calibration of the sensor response function from a sequence of images affected by the limited dynamic range of the camera. The technique requires only as input a set of differently exposed fringe patterns. In this case, the different integration times are not necessary as inputs of the algorithm.

\section{Proposed method}

The proposed method first obtains for each single-exposed fringe pattern its normalized version, $I_{n, j}(x, y)$ in expression (2), and the modulation map, $I_{m, j}(x, y)$ in expression (1) using the method shown in [6]. Note that the sub-index $j$ denotes the differently exposed fringe patterns and $j=[1, N]$, with $N$ the number of differently exposed fringe patterns. Note that we have used the normalization method

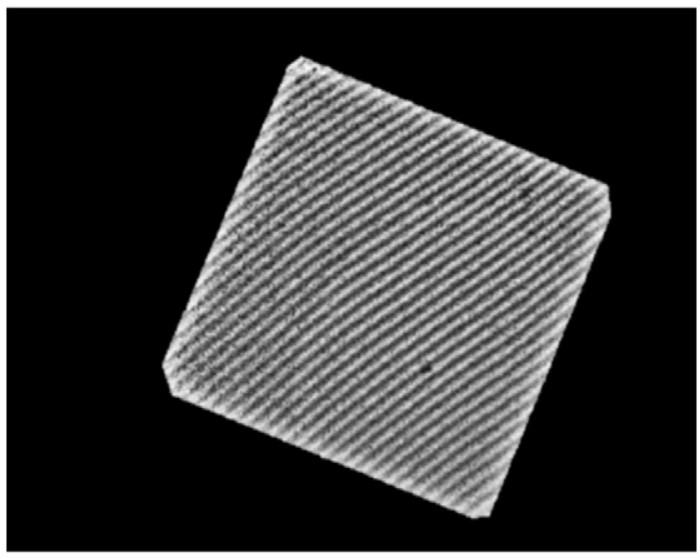

Fig. 3. Resultant extended dynamic range fringe pattern. 


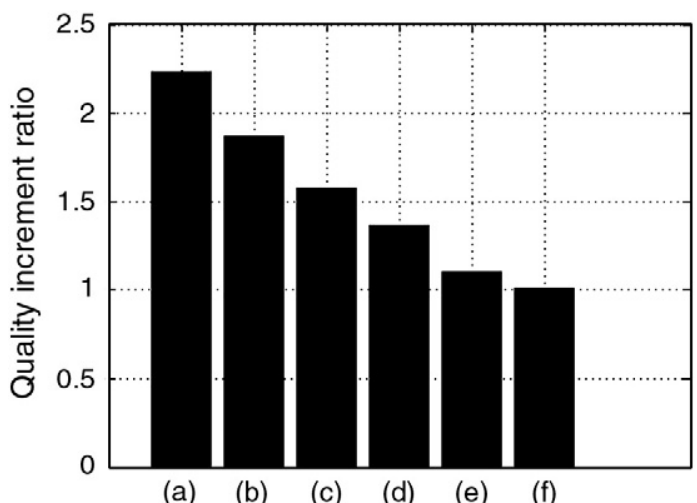

(a) $(c) \quad(d) \quad(e)$
Fringe pattern

Fig. 4. Quality increment of $I_{e}$ with respect to the patterns shown in Fig. 1.

Table 1

Quality increment ratio obtained in the first experiment.

\begin{tabular}{ll}
\hline (a) & 2.24 \\
(b) & 1.87 \\
(c) & 1.57 \\
(d) & 1.36 \\
(e) & 1.10 \\
(f) & 1.01 \\
\hline
\end{tabular}

shown in [6] but any normalization approach can be used. The modulation map is an important magnitude to measure the quality of a fringe pattern [7]. For each single exposed pattern, in the regions where the modulation map presents large values, the fringe pattern is not overexposed neither underexposed. In the opposite case, if the modulation map has low values the corresponding fringe pattern is not reliable at these points. Note that the maximum and minimum modulation values in a typical camera with a dynamic range of 256

gray levels are 255 and 0 respectively so the terms large and low are referred to these values. Once we have the sequence of the normalized fringe patterns $I_{n, j}$ and the corresponding modulation maps, $I_{m, j}$ we can compose the HDR fringe pattern $\left(I_{e}\right)$ as a temporal weighted average using as weights the corresponding modulation maps,

$I_{e}(x, y)=\frac{\sum_{j=1}^{N} I_{n, j}(x, y) I_{m, j}(x, y)}{\sum_{j=1}^{N} I_{m, j}(x, y)}$.

In expression (3), in the regions where the denominator is equal to zero we impose that $I_{e}(x, y)=0$.

\section{Experimental results}

We have checked the proposed algorithm with experimental interferograms obtained by a Mach-Zenhder interferometer. In the first experiment we check our proposed method with experimental open-fringe patterns. In Figs. 1 and 2 we show six interferograms obtained with different unknown integration times and their corresponding obtained modulation maps using the method shown in [7]. The size of each image is $1280 \times 960$.

In order to quantify the increment of quality of the HDR fringe pattern $I_{e}$ (Fig. 3) with respect to the different exposed fringe patterns, we obtain the modulation map of the HDR interferogram $\left(I_{m, e}\right)$ using again the method shown in [7] and then we use the following expression,

$Q_{j}=\left\langle\frac{I_{m, e}(x, y)}{I_{m}, j(x, y)}\right\rangle$

where, $Q_{j}$ denotes the fringe quality increment ratio of $I_{e}$ with respect to $I_{j}$ and $<>$ is the spatial average. If $Q_{j} \gg 1$ then the increment of quality is large. If $Q_{j}=1$ there is no quality increase. In Fig. 4 and Table 1 we show the values of $Q_{j}$ obtained in the first experiment. As can be seen from Fig. 4 and Table $1 Q$ is larger than one in all cases. In the second experiment we check the proposed method (a)

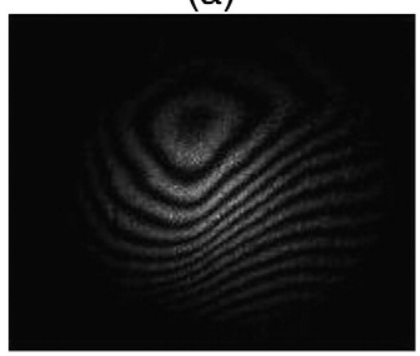

(d)

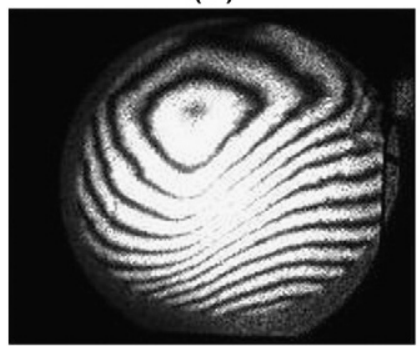

(b)

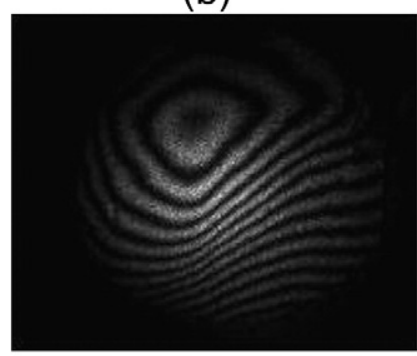

(e)

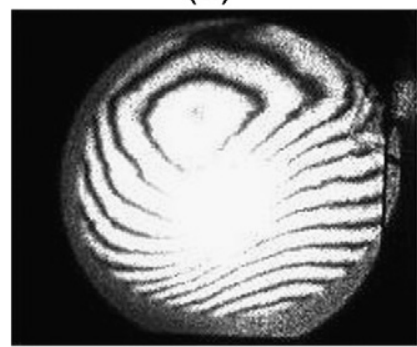

(c)
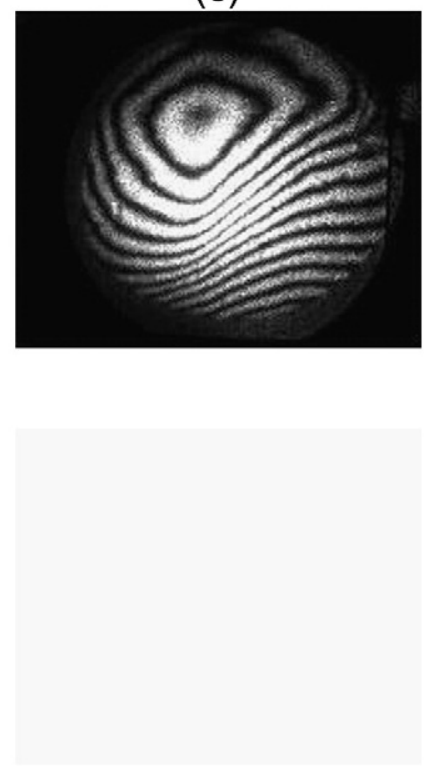

Fig. 5. Differently exposed closed fringe pattern images used in the second experiment. 
(a)

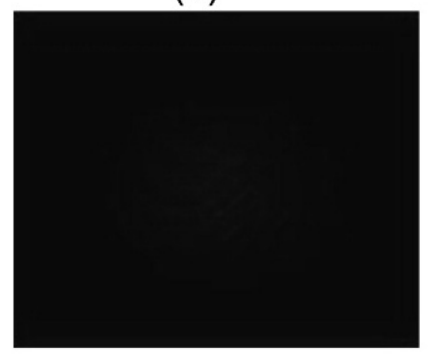

(d)

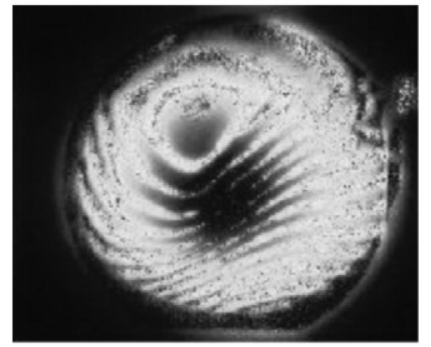

(b)

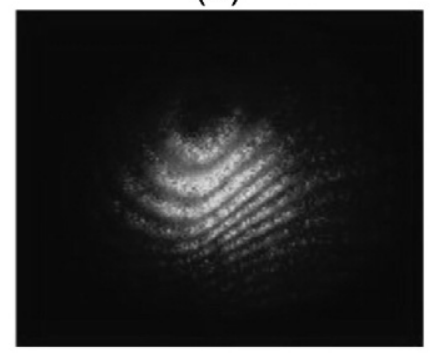

(e)

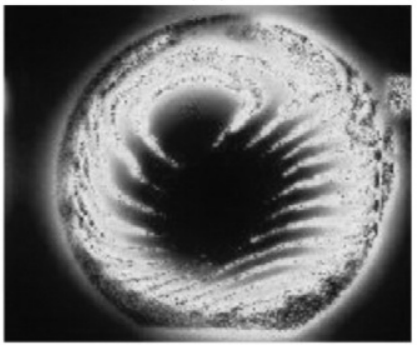

(c)

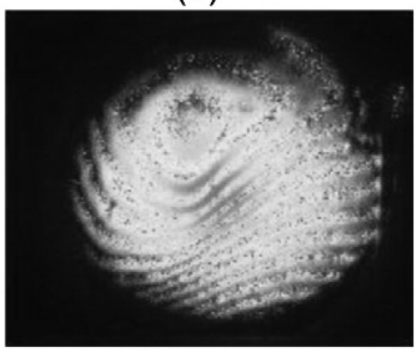

Fig. 6. Modulation maps corresponding to the fringe patterns shown in Fig. 5.

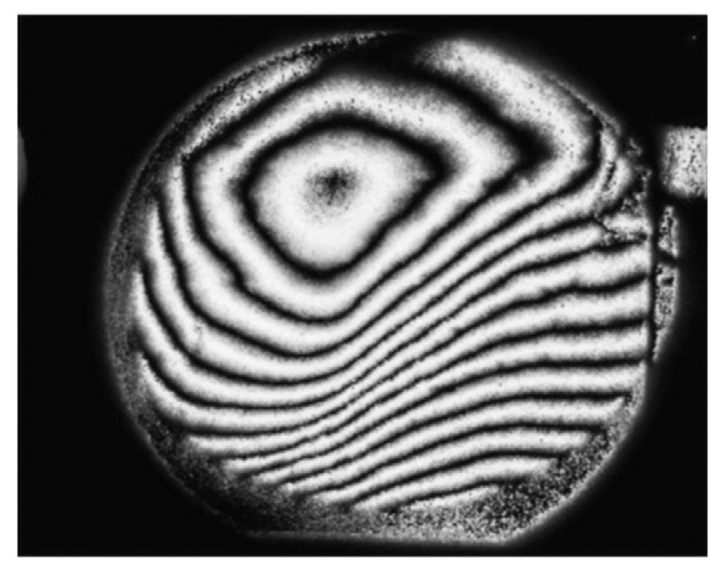

Fig. 7. Resultant extended dynamic range fringe pattern.

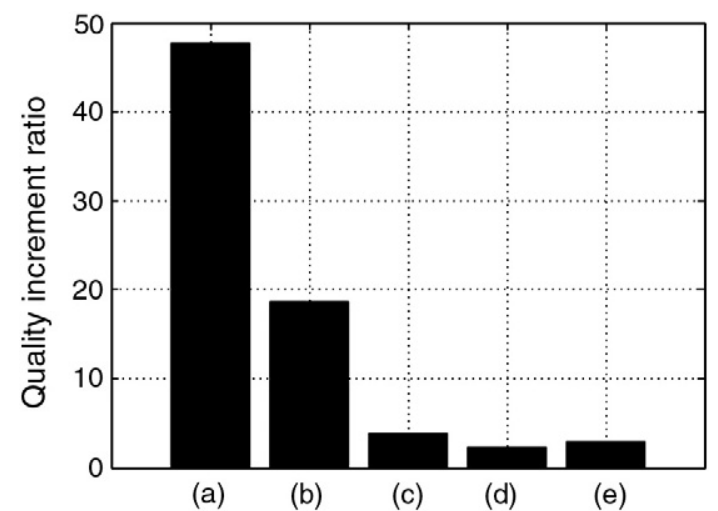

Fringe pattern
Table 2

Quality increment ratio obtained in the second experiment.

\begin{tabular}{lr}
\hline (a) & 49 \\
(b) & 19 \\
(c) & 4 \\
(d) & 2 \\
(e) & 3 \\
\hline
\end{tabular}

with experimental closed-fringe patterns. In Fig. 5 we show five interferograms obtained with different unknown integration times. As can be seen in Fig. 5 the interferograms present very bright and dark regions. In Fig. 6 we show the obtained modulation maps using [7] as in the previous experiment. From Figs. 5 and 6 it is possible to see that if the camera exposure time is low then the dark regions are lost in sensor noise. On the other hand if this time is high then the bright regions are overexposed. In both cases, the modulation map presents low values and it is not possible to acquire reliable information from these fringe patterns at these pixels. In Fig. 7 we show the resultant HDR fringe pattern. In Fig. 8 and Table 2 we show the fringe quality increment ratio $Q_{j}$ of $I_{e}$ with respect to the single exposed fringe patterns shown in Fig. 5. As can be seen from Figs. 4 and 8 and Tables 1 and 2, the increment of quality is significantly higher in the second experiment. This is because the modulation distribution is considerably more inhomogeneous in the interferograms of the second experiment than in the ones of the first experiment.

\section{Conclusions}

In this work we propose a HDR method specially designed for interferometry. The proposed method doesn't need to know the different exposure times or calibrating the camera response curve as standard HDR techniques. We have shown experimental results that show the efficiency of the proposed technique. All the examples of this work can be reproduced running the MATLAB package that can be found in [8].

Fig. 8. Increment of quality of $I_{e}$ with respect to the different single exposed fringe pattern shown in Fig. 5. 


\section{References}

11] D. Malacara, M. Servín, Z. Malacara, Interferogram Analysis for Optical Testing Marcel Dekker Inc, 1998.

[2] P.E. Debevec, J. Malik, Recovering high dynamic range radiance maps from photographs, SIGGRAPH, 97, 1997, p. 369.

[3] J. Vargas, J.A. Quiroga, T. Koninckx, L. Van Gool, Opt. Eng 47 (5) (2008).
[4] J. Vargas, L. González-Fernandez, J. Antonio Quiroga, T. Belenguer, Appl. Opt. 49 (2010) 2409.

[5] Roger M. Groves, Pedrini Giancarlo, Osten Wolfgang, Appl. Opt. 47 (2008) 5550.

6] J.A. Quiroga, M. Servín, Opt. Commun. 224 (2003) 221.

7] B. Strobel, Appl. Opt. 35 (13) (1996) 2192

[8] http://goo.gl/4y1Ql. 